Stud. Univ. Babeş-Bolyai Math. 64(2019), No. 2, 253-262

DOI: 10.24193/subbmath.2019.2.10

\title{
Stone-Weierstrass theorems for random functions
}

\author{
Hans-Jörg Starkloff
}

\begin{abstract}
We present several generalizations of the Stone-Weierstrass theorem concerning the approximation of continuous functions on a compact set by using functions from a subalgebra to the case of random functions and random variables in the space of continuous functions. The continuity of the random functions is allowed to be only with respect to a metric, hence including the case of stochastically continuous random functions. These results could be cornerstones for the general theory of approximation for random functions.
\end{abstract}

Mathematics Subject Classification (2010): 41A65, 60G07, 60B11.

Keywords: Stone-Weierstrass theorem, approximation of random functions, stochastic convergence, random polynomial.

\section{Introduction}

It is well known that approximation theory plays an important role in the mathematical investigation of deterministic equations and for other problems. Therefore it seems to be natural that approximation theory should also play a similar important role in the investigation of different types of random equations. Moreover in recent time an increased use of stochastic models and a very intensive investigation of random and stochastic differential equations can be observed. So the systematic investigation of approximation procedures and possibilities for random mathematical objects seems to be useful, leading possibly to the development of an approximation theory for random functions and random variables in function spaces.

One fundamental result in deterministic approximation theory is the StoneWeierstrass theorem about the uniform closure of a subalgebra of continuous functions in the space of all continuous functions on a compact set. This result is a generalization of the classical theorem of Weierstrass about the denseness (with respect to the norm of uniform convergence) of polynomials in the space of continuous functions on a closed interval of the real line.

This paper has been presented at the fourth edition of the International Conference on Numerical Analysis and Approximation Theory (NAAT 2018), Cluj-Napoca, Romania, September 6-9, 2018. 
The presented work deals with analogous questions for random functions. Hereby different generalizations can be considered, due for example to the use of different norms or metrics in spaces of random variables.

In the remaining part of this section a short remainder on deterministic StoneWeierstrass theorems together with some needed definitions is given. The following section is devoted to different theorems of Stone-Weierstrass type for random functions or random variables with values in function spaces.

The following notations and concepts are used throughout the article.

- $T$ denotes a compact Hausdorff topological space.

- $\mathbb{K}=\mathbb{R}$ or $\mathbb{K}=\mathbb{C}$ denotes the scalar field (of real or complex numbers), $\mathbb{N}^{*}=\{1,2,3, \ldots\}$ is the set of positive natural numbers.

- $\mathrm{C}(T, \mathbb{K})$ denotes the linear space of continuous $\mathbb{K}$-valued functions on $T$, endowed with the maximum norm (this way it becomes a Banach space and moreover a Banach algebra).

- $\mathbf{1}_{S}$ denotes the indicator function of the set $S$ (also called characteristic function) with values 1 for elements from $S$ and 0 otherwise.

- A set $A$ of $\mathbb{K}$-valued functions on $T$ is called an algebra if it holds

$$
f, g \in A, \alpha \in \mathbb{K} \Rightarrow f+g \in A, \alpha f \in A, f \cdot g \in A .
$$

The classical theorem of Weierstrass about the approximation of real continuous functions by algebraic polynomials can be formulated as follows.

Theorem 1.1. The set (algebra) of polynomials with real coefficients is dense in the space of real-valued continuous functions on a finite interval $[a, b] \subset \mathbb{R}$, endowed with the maximum norm.

Analogous results hold for complex-valued functions and for functions defined on $\left[a_{1}, b_{1}\right] \times \ldots \times\left[a_{d}, b_{d}\right] \subset \mathbb{R}^{d}\left(d \in \mathbb{N}^{*}\right)$.

The approximability of continuous functions by algebraic polynomials is strongly related to the approximability of periodic functions by trigonometric polynomials.

Theorem 1.2. The set (algebra) of trigonometric polynomials with real coefficients is dense in the space of real-valued $2 \pi$-periodic continuous functions on the interval $[0,2 \pi] \subset \mathbb{R}$, endowed with the maximum norm.

Analogous results hold for complex-valued functions, for periodic functions defined on $[0,2 \pi]^{d} \subset \mathbb{R}^{d}\left(d \in \mathbb{N}^{*}\right)$ and for periodic functions with other periods.

One generalization of this result, namely a version of the theorem of StoneWeierstrass can be given as follows.

Theorem 1.3. A subalgebra $A \subset \mathrm{C}(T, \mathbb{K})$ is dense in $\mathrm{C}(T, \mathbb{K})$, if and only if

(i) A separates the points of $T$ ( $A$ is point-separating on $T)$, i.e.,

$$
\forall t_{1} \neq t_{2} \in T \exists f \in A: f\left(t_{1}\right) \neq f\left(t_{2}\right) ;
$$

(ii) $A$ is not point-vanishing on $T$, i.e., $\forall t \in T \exists f \in A: f(t) \neq 0$;

(iii) $A$ is self-adjoint in the case $K=\mathbb{C}$, i.e., $\forall f \in A \Rightarrow \bar{f} \in A$. 
These theorems can be found in many textbooks for analysis or approximation theory. Other versions of the Stone-Weierstrass theorem, different proofs, etc. can be found for example in Prolla [6].

\section{Stone-Weierstrass theorems for random functions and random variables with values in a function space}

In the following all random objects are assumed to be defined on a probability space $(\Omega, \mathcal{F}, \mathrm{P})$. The corresponding expectation operator is denoted by $\mathrm{E}^{0} \mathrm{~L}^{0}(\mathbb{K})$ denotes the linear space of equivalence classes of random variables with values in $\mathbb{K}$ (and analogously for other ranges). For $\mathbb{K}$-valued random variables the stochastic convergence is connected to the concept of metric, one possible metric is

$$
d_{p}\left(\eta_{1}, \eta_{2}\right)=\mathrm{E}\left[\frac{\left|\eta_{1}-\eta_{2}\right|}{1+\left|\eta_{1}-\eta_{2}\right|}\right], \quad \eta_{1}, \eta_{2} \in \mathrm{L}^{0}(\mathbb{K}),
$$

with analogous expression in the case of a (semi-)normed space as range instead of $\mathbb{K}$. As usual $L^{p}(\mathbb{K})$ is the linear space of equivalence classes of $\mathbb{K}$-valued random variables with finite moment of order $p$, endowed with the corresponding norm $(1 \leq p<\infty)$ or metric $(0<p<1)$.

Stochastic generalizations of the Stone-Weierstrass theorem can be derived

- for random variables (random elements) with values in $\mathrm{C}(T, \mathbb{K})$ or

- for $\mathbb{K}$-valued random functions $\left(\xi_{t}\right)_{t \in T}$, continuous in some sense.

Further generalizations can be given for example for random functions with values in separable Banach spaces.

The approximating set is often the linear span (which is not necessarily an algebra)

$$
\operatorname{span}\left\{\eta f: \eta \in V \subseteq \mathrm{L}^{0}(\mathbb{K}), f \in A \subseteq \mathrm{C}(T, \mathbb{K})\right\}
$$

with suitable sets $V$ and $A$.

The notion or type of convergence for (generalized) sequences of random variables or random functions can be generated by norms or metrics or otherwise. In case of norms many theorems can be generalized straightforwardly. More difficult is the study of these results and definitions in the context of a metric (which is for example valid for the case of stochastic convergence).

As a first generalization of the deterministic Stone-Weierstrass theorem a result for random variables in the space of continuous functions and stochastic convergence is given.

Theorem 2.1. Assume that $\mathbb{X}$ is a separable Banach space with norm $\|\cdot\|$ or more generally a complete separable metrizable locally convex space with metric $\|\cdot\|_{\mathbb{X}}$ over the field $\mathbb{K}, T$ is a compact metric space with metric $r$ and $A \subseteq \mathrm{C}(T, \mathbb{K})$ is a selfadjoint algebra, which is point-separating and not point-vanishing on $T$. Then

$$
S:=\operatorname{span}\left\{\eta f: \eta \in \mathrm{L}^{0}(\mathbb{X}), f \in A\right\}
$$


is dense with respect to the stochastic convergence in $\mathrm{L}^{0}(\mathrm{C}(T, \mathbb{X}))$, i.e.,

$$
\forall \varepsilon>0 \forall \xi \in \mathrm{L}^{0}(\mathrm{C}(T, \mathbb{X})) \exists \zeta \in S: \mathrm{P}\left(\max _{t \in T}\left\|\xi_{t}-\zeta_{t}\right\|_{\mathbb{X}}>\varepsilon\right)<\varepsilon .
$$

Proof. Convergence in a complete separable metrizable locally convex space is equivalent to the convergence for each of an at most countable defining system of half-norms (see, e.g. Rudin [9], Theorem 1.24, Remarks 1.38). So the proof is given for the case of a separable Banach space $\mathbb{X}$ with norm $\|\cdot\|$ and it is identical for each of the half-norms in the locally convex spaces.

In this case the asserted property is equivalent to

$$
\forall \varepsilon>0 \forall \xi \in \mathrm{L}^{0}(\mathrm{C}(T, \mathbb{X})) \exists \zeta \in S: \mathrm{E}\left[\frac{\max _{t \in T}\left\|\xi_{t}-\zeta_{t}\right\|}{1+\max _{t \in T}\left\|\xi_{t}-\zeta_{t}\right\|}\right]<\varepsilon .
$$

$\mathrm{C}(T, \mathbb{X})$ is separable if $T$ is a compact metric space. Hence for each $\varepsilon>0$ there exists a compact set $K \subset \mathrm{C}(T, \mathbb{X})$ with $\mathrm{P}(\xi \in K)>1-\varepsilon / 3$ (see, e.g. Billingsley [1], Theorem $1.4)$.

Define $\xi^{\prime}:=\xi \cdot \mathbf{1}_{\{\xi \in K\}}$, so that $\mathrm{P}\left(\max _{t \in T}\left\|\xi_{t}-\xi_{t}^{\prime}\right\|>0\right)<\varepsilon / 3$ and hence

$$
\mathrm{E}\left[\frac{\max _{t \in T}\left\|\xi_{t}-\xi_{t}^{\prime}\right\|}{1+\max _{t \in T}\left\|\xi_{t}-\xi_{t}^{\prime}\right\|}\right]<\varepsilon / 3
$$

The functions in $K$ and hence the realizations of $\xi^{\prime}$ are bounded and equicontinuous (see, e.g., Rudin [9], Theorems A 4, A 5, Dieudonné [3], Theorem 7.5.7). Hence there exists $\delta>0$ with $\left\|\xi_{t}^{\prime}(\omega)-\xi_{s}^{\prime}(\omega)\right\|<\varepsilon / 3$ for all $\omega \in \Omega$ if $r(t, s)<\delta$. The compact set $T$ can be covered by a finite number of $\delta$-neighbourhoods of points $t_{i} \in T, i=1, \ldots, n$. Assume $\left(z_{i}, i=1, \ldots, n\right)$ is a partition of unity subordinate to this open cover (see, e.g., Rudin [8], Theorem 2.13). Defining

$$
\hat{\xi}_{t}:=\sum_{i=1}^{n} \xi_{t_{i}}^{\prime} z_{i}(t), \quad t \in T
$$

it holds for arbitrary $t \in T$ and $\omega \in \Omega$

$$
\left\|\hat{\xi}_{t}(\omega)-\xi_{t}^{\prime}(\omega)\right\| \leq \sum_{i=1}^{n}\left\|\xi_{t_{i}}^{\prime}-\xi_{t}^{\prime}\right\| z_{i}(t) \leq \sum_{i=1}^{n} \frac{\varepsilon}{3} z_{i}(t)=\frac{\varepsilon}{3} .
$$

This means that the set $\operatorname{span}\left\{\eta f: \eta \in \mathrm{L}^{0}(\mathbb{X}), f \in \mathrm{C}(T, \mathbb{K})\right\}$ is dense with respect to the stochastic convergence in $\mathrm{L}^{0}(\mathrm{C}(T, \mathbb{X}))$. It remains to approximate the functions $z_{i} \in \mathrm{C}(T, \mathbb{K})$ uniformly by functions from $A \subseteq \mathrm{C}(T, \mathbb{K})$, which is possible using the deterministic Stone-Weierstrass Theorem 1.3.

Corollary 2.2. Assume that $T$ is a compact metric space with metric $r$ and $A \subseteq$ $\mathrm{C}(T, \mathbb{K})$ is a self-adjoint algebra, which is point-separating and not point-vanishing on $T$. Then $S:=\operatorname{span}\left\{\eta f: \eta \in \mathrm{L}^{0}(\mathbb{K}), f \in A\right\}$ is dense with respect to the stochastic convergence in $\mathrm{L}^{0}(\mathrm{C}(T, \mathbb{K}))$, i.e.,

$$
\forall \varepsilon>0 \forall \xi \in \mathrm{L}^{0}(\mathrm{C}(T, \mathbb{K})) \exists \zeta \in S: \mathrm{P}\left(\max _{t \in T}\left|\xi_{t}-\zeta_{t}\right|>\varepsilon\right)<\varepsilon .
$$

Remark 2.3. In relation to the above theorem one can remark the following. 
- One can find for every $\xi \in \mathrm{L}^{0}(\mathrm{C}(T, \mathbb{X}))$ a sequence $\left(\zeta_{n}\right)_{n \in \mathbb{N}^{*}} \subset S$, which converges almost surely to $\xi$.

- Analogous results hold for $\mathrm{L}^{p}(\mathrm{C}(T, \mathbb{X}))(1 \leq p<\infty)$ with convergence in $p$-th mean.

- $\mathrm{C}(T, \mathbb{K})$ is separable iff $T$ is a compact metric space (see, e.g., Rolewicz [7], Proposition 1.6.6). The investigation of random variables with values in nonseparable normed spaces is much more complicated, so we restrict ourselves to the case of a separable Banach space $\mathrm{C}(T, \mathbb{K})$.

Now we consider the case of $\mathbb{K}$-valued random functions $\left(\xi_{t}\right)_{t \in T}$ which are continuous in some sense. Correspondig results can be found in the literature for example in Dugué [4] ( $T \subset \mathbb{R}$ finite interval, approximation of a random function $\left(\xi_{t}\right)_{t \in T}$, which is continuous in probability, by random polynomials with respect to the uniform stochastic convergence), Onicescu, Istrăţescu [5] $\left(T \subset \mathbb{R}^{d}\left(d \in \mathbb{N}^{*}\right)\right.$ convex compact set, uniform stochastic approximation by multivariate random polynomials) and Ryabykh, Tokmakova, Yablonski $\breve{i 0]}\left(T \subset \mathbb{R}^{d}\left(d \in \mathbb{N}^{*}\right)\right.$ compact set, uniform stochastic approximation by elements of a subalgebra of random functions). It can be remarked, that, in general, spaces of random variables endowed with the topology of stochastic convergence are not locally convex spaces, so that generalizations of Stone-Weierstrass theorems for locally convex spaces (see, e.g. Timofte [11]) are not applicable in this situation.

A stochastic generalization of Stone-Weierstrass theorem is easy to obtain if a topology in the space of random variables induced by a norm is used.

Theorem 2.4. Assume $(V,\|\cdot\|)$ is a normed subspace of $\mathrm{L}^{0}(\mathbb{K})$ and $A \subseteq \mathrm{C}(T, \mathbb{K})$ is a self-adjoint algebra, which is point-separating and not point-vanishing on the compact Hausdorff topological space T. Then

$$
S:=\operatorname{span}\{\eta f: \eta \in V, f \in A\}
$$

is dense in $\mathrm{C}(T, V)$ with respect to the uniform $\|\cdot\|$-convergence on $T$.

Proof. For $\xi \in \mathrm{C}(T, V)$ and $\varepsilon>0$ consider the open cover of $T$ defined by sets $U_{t}:=\left\{s \in T:\left\|\xi_{s}-\xi_{t}\right\|<\varepsilon / 2\right\}$. Due to the compactness one can choose a finite open subcover of $T$ with sets $U_{t_{i}}$, where $t_{i} \in T, i=1, \ldots, n \in \mathbb{N}^{*}$, are points which are pairwise disjoint. Assume $\left(z_{i}, i=1, \ldots, n\right)$ is a partition of unity subordinate to this open subcover and define

$$
\hat{\xi}_{t}:=\sum_{i=1}^{n} \xi_{t_{i}} z_{i}(t), \quad t \in T .
$$

Then it holds $\hat{\xi} \in \operatorname{span}\{\eta f: \eta \in V, f \in \mathrm{C}(T, \mathbb{K})\}$ and for all $t \in T$

$$
\left\|\hat{\xi}_{t}-\xi_{t}\right\| \leq \sum_{i=1}^{n}\left\|\xi_{t_{i}}-\xi_{t}\right\| z_{i}(t)<\varepsilon / 2,
$$

because it holds $z_{i}(t)>0$ only for points $t \in U_{t_{i}}$. It remains to approximate the functions $z_{i} \in \mathrm{C}(T, \mathbb{K})$ uniformly by functions $\tilde{z}_{i} \in A \subseteq \mathrm{C}(T, \mathbb{K})$, which is possible by 
the deterministic Stone-Weierstrass Theorem 1.3, in such a way that

$$
\max _{t \in T}\left|z_{i}(t)-\tilde{z}_{i}(t)\right|<\varepsilon /(2 n M), \quad i=1, \ldots, n,
$$

where $M:=\max _{t \in T}\left\|\xi_{t}\right\|<\infty$. Then for

$$
\tilde{\xi}_{t}:=\sum_{i=1}^{n} \xi_{t_{i}} \tilde{z}_{i}(t), \quad t \in T,
$$

it holds $\tilde{\xi} \in \operatorname{span}\{\eta f: \eta \in V, f \in A\}$ and for all $t \in T$

$$
\left\|\tilde{\xi}_{t}-\hat{\xi}_{t}\right\| \leq \sum_{i=1}^{n}\left\|\xi_{t_{i}}\right\| \cdot\left|\tilde{z}_{i}(t)-z_{i}(t)\right|<\varepsilon / 2 .
$$

Now from the triangle inequality the assertion follows.

This result is valid for arbitrary functions with values in a (half-)normed space, defined on a compact set and can be deduced easily also for example from Theorem 1 in Chapter 2 of Prolla [6].

Measuring the nearness of random variables with the help of a metric it is desirable that $V \subseteq \mathrm{L}^{0}(\mathbb{K})$ is a metric linear space, i.e., the linear operations are continuous. Then from general theory it follows (see, e.g., Rolewicz [7], Theorems 1.1.1 and 1.2.2) that there exists a translation-invariant non-decreasing metric (an " $F$-norm" denoted by $\|\cdot\|)$, which is equivalent to the given metric. Basic properties of an $F$-norm on $\mathrm{L}^{0}(\mathbb{K})$ are

(F 1) $\left\|\mathbf{1}_{\Omega}\right\|<\infty$.

(F 2) $\|\xi\|=0 \Leftrightarrow \xi=0$ a. s.

(F 3) $\|\alpha \xi\| \leq\|\xi\|$ for all $\alpha \in \mathbb{K}$ with $|\alpha| \leq 1$.

(F 4) $\|\xi+\eta\| \leq\|\xi\|+\|\eta\|$.

(F 5) $\left\|a_{n} \xi\right\| \rightarrow 0$, if $a_{n} \rightarrow 0 \quad(n \rightarrow \infty)$.

In certain cases further properties of the $F$-norm are imposed.

(F 6) $|\xi| \leq|\eta|$ a.s. and $\|\eta\|<\infty \Rightarrow\|\xi\| \leq\|\eta\|$.

(F 7) For each sequence $\left(F_{n}\right)_{n \in \mathbb{N}^{*}}, F_{n} \in \mathcal{F}$ with $\mathrm{P}\left(F_{n}\right) \rightarrow 0$ and every $\xi \in \mathrm{L}^{0}(\mathbb{K})$ with $\|\xi\|<\infty$ it holds $\left\|\xi \mathbf{1}_{F_{n}}\right\| \rightarrow 0 \quad(n \rightarrow \infty)$.

(F 8) There exists a constant $\kappa=\kappa(c)>0$ such that for arbitrary random variables $\xi$ with $\mathrm{P}(|\xi| \leq c)=1$ for a real number $c>0$ and for arbitrary $a \in \mathbb{R}$ it holds

$$
\|a \xi\| \leq|a| \kappa(c)\|\xi\| \text {. }
$$

The $F$-norm generating the stochastic convergence is $\|\xi\|_{p}=d_{p}(\xi, 0)$ and fulfills all the properties (F 1)-(F 7) from above and also (F 8) as is proven below in Lemma 2.9 .

Some further properties related to $F$-norms are stated now.

Lemma 2.5. Let $(V,\|\cdot\|)$ be an F-normed subspace of $\mathrm{L}^{0}(\mathbb{K})$, such that the basic and the additional properties (F 1)-(F 7) of F-norms are fulfilled and let $\left(\xi_{n}\right)_{n \in \mathbb{N}}$ be a sequence of elements from $V$ which converges stochastically to $\xi \in V$ and such that for a random variable $\eta \in V$ it holds $\mathrm{P}\left(\left|\xi_{n}\right| \leq \eta\right)=1$ for all $n \in \mathbb{N}$. Then it holds also $\left\|\xi_{n}-\xi\right\| \rightarrow 0$ for $n \rightarrow \infty$. 
Proof. One shows that from each subsequence $\left(\xi_{n^{\prime}}\right)$ a subsubsequence $\left(\xi_{n^{\prime \prime}}\right),\left\{n^{\prime \prime}\right\} \subseteq$ $\left\{n^{\prime}\right\}$, can be chosen such that $\left\|\xi_{n^{\prime \prime}}-\xi\right\| \rightarrow 0$ for $n^{\prime \prime} \rightarrow \infty$. Then the assertion follows.

Due to the stochastic convergence of $\left(\xi_{n^{\prime}}\right)$ to $\xi$ one can choose a subsubsequence $\left(\xi_{n^{\prime \prime}}\right)$ which converges almost surely to $\xi$. By the Theorem of Egorov (see, e.g. Bogachev [2], Theorem 2.2.1) it follows that for every $\varepsilon>0$ there exists $B_{\varepsilon} \in \mathcal{F}$ with $\mathrm{P}\left(\Omega \backslash B_{\varepsilon}\right)<$ $\varepsilon$ and uniform convergence of $\left(\xi_{n^{\prime \prime}}\right)$ to $\xi$ on $B_{\varepsilon}$. In

$$
\left\|\xi_{n^{\prime \prime}}-\xi\right\| \leq\left\|\left(\xi_{n^{\prime \prime}}-\xi\right) \mathbf{1}_{B_{\varepsilon}}\right\|++\left\|\left(\xi_{n^{\prime \prime}}-\xi\right) \mathbf{1}_{\Omega \backslash B_{\varepsilon}}\right\|
$$

the first summand on the right hand side converges for each $\varepsilon>0$ for $n^{\prime \prime} \rightarrow \infty$ to zero due to the uniform convergence and properties (F 5) and (F 6). From property (F 7) it follows hat the second summand on the right hand side converges for $\varepsilon \rightarrow 0$ to zero.

Corollary 2.6. Let $(V,\|\cdot\|)$ be an $F$-normed subspace of $\mathrm{L}^{0}(\mathbb{K})$, such that the basic and the additional properties (F 1)-(F 7) of F-norms are fulfilled and let $T$ be a set. Assume $\left(\xi_{t}^{n}, t \in T\right)_{n \in \mathbb{N}}$ is a sequence of functions with values in $V$ such that there exists $\eta \in V$ with $\mathrm{P}\left(\left|\xi_{t}^{n}\right| \leq \eta\right)=1$ for all $t \in T$ and $n \in \mathbb{N}$. Then it holds for a random function $\left(\xi_{t}\right)_{t \in T}$ with values in $V$

$$
\lim _{n \rightarrow \infty} \sup _{t \in T}\left\|\xi_{t}^{n}-\xi_{t}\right\|_{p}=0 \Rightarrow \lim _{n \rightarrow \infty} \sup _{t \in T}\left\|\xi_{t}^{n}-\xi_{t}\right\|=0 .
$$

Proof. Denote $V_{\eta}=\{\xi \in V:|\xi| \leq \eta\}$. The assertion follows from the fact that the identity operator from $\left(V_{\eta},\|\cdot\|_{p}\right)$ to $\left(V_{\eta},\|\cdot\|\right)$ is continuous by Lemma 2.5.

Lemma 2.7. Let $(V,\|\cdot\|)$ be an F-normed subspace of $\mathrm{L}^{0}(\mathbb{K})$, such that the basic properties (F 1)-(F 5) of F-norms are fulfilled, $\xi \in V$ and let be given $f_{n} \in \mathrm{C}(T, \mathbb{K})$, $n \in \mathbb{N}^{*}$, such that $f_{n} \rightarrow f(n \rightarrow \infty)$ in $\mathrm{C}(T, \mathbb{K})$. Then it holds also

$$
\lim _{n \rightarrow \infty} \sup _{t \in T}\left\|\xi\left(f_{n}(t)-f(t)\right)\right\|=0 .
$$

Proof. This follows directly from property (F 5) and the assumptions.

For a real-valued random variable $\xi$ and a real number $c>0$ its truncation is defined by $\xi^{(c)}:=\sup \{-c, \inf \{\xi, c\}\}$ (and analogously for the real and imaginary part in the complex-valued case). So it follows that $\mathrm{P}\left(\left|\xi^{(c)}\right| \leq c\right)=1$.

Lemma 2.8. Assume $T$ is a compact Hausdorff topological space, $(V,\|\cdot\|)$ is an $F$-normed subspace of $\mathrm{L}^{0}(\mathbb{K})$, such that the basic and the additional properties $(\mathrm{F} 1)$ (F 7) of F-norms are fulfilled and such that $\forall c>0 \xi \in V \Rightarrow \xi^{(c)} \in V$. Furthermore let $\xi=\left(\xi_{t}\right)_{t \in T}$ be a continuous function with values in $V$.

(i) $\left(\xi_{t}^{(c)}\right)_{t \in T}$ is continuous in $(V,\|\cdot\|)$ for arbitrary $c>0$.

(ii) $\lim _{c \rightarrow \infty} \sup _{t \in T}\left\|\xi_{t}^{(c)}-\xi_{t}\right\|=0$.

Proof. (i) The assertion follows from property (F 6).

(ii) For $\xi \in \mathrm{C}(T, V)$ and $\varepsilon>0$ consider the open cover of $T$ defined by sets

$$
U_{t}:=\left\{s \in T:\left\|\xi_{s}-\xi_{t}\right\|<\varepsilon / 3\right\} .
$$

Due to the compactness one can choose a finite open subcover of $T$ with sets $U_{t_{i}}$, where $t_{i} \in T, i=1, \ldots, n \in \mathbb{N}^{*}$, are points which are pairwise disjoint. Then for each 
$t \in T$ one has $\left\|\xi_{t_{i}}-\xi_{t}\right\|<\varepsilon / 3$ for one $t_{i}$ and consequently by property (F 6 ) also $\left\|\xi_{t_{i}}^{(c)}-\xi_{t}^{(c)}\right\|<\varepsilon / 3$ for arbitrary $c>0$. Property (F 7) allows us to find $c>0$ such that for all $i=1, \ldots, n$ it holds $\left\|\xi_{t_{i}}^{(c)}-\xi_{t_{i}}\right\|<\varepsilon / 3$. Then it follows

$$
\left\|\xi_{t}^{(c)}-\xi_{t}\right\| \leq\left\|\xi_{t}^{(c)}-\xi_{t_{i}}^{(c)}\right\|+\left\|\xi_{t_{i}}^{(c)}-\xi_{t_{i}}\right\|+\left\|\xi_{t_{i}}-\xi_{t}\right\|<\varepsilon
$$

Lemma 2.9. Let $\xi$ be a random variable with $\mathrm{P}(|\xi| \leq c)=1$ for a real number $c>0$. Then $\|\cdot\|_{p}$ fulfills (F 8), in particular it holds for arbitrary $a \in \mathbb{R}$

$$
\|a \xi\|_{p} \leq|a|(c+1)\|\xi\|_{p}
$$

Proof. It holds

and

$$
\|a \xi\|_{p}=\mathrm{E}\left[\frac{|a \xi|}{1+|a \xi|}\right] \leq|a| \mathrm{E}\left[\frac{1+|\xi|}{1+|a \xi|} \frac{|\xi|}{1+|\xi|}\right]
$$

$$
\mathrm{P}\left(\frac{1+|\xi|}{1+|a \xi|} \leq 1+c\right)=1 \text {. }
$$

Theorem 2.10. Assume $T$ is a compact Hausdorff topological space, $(V,\|\cdot\|)$ is an $F$-normed subspace of $\mathrm{L}^{0}(\mathbb{K})$, such that the basic and the additional properties ( $\left.\mathrm{F} 1\right)$ (F 8) of $F$-norms are fulfilled and $A \subseteq \mathrm{C}(T, \mathbb{K})$ is a self-adjoint algebra, which is pointseparating and not point-vanishing on $T$. Then the set $S:=\operatorname{span}\{\eta f: \eta \in V, f \in A\}$ is dense with respect to the uniform $\|\cdot\|$-convergence on $T$, i.e., in $\mathrm{C}(T, V)$.

Proof. First it is proved that $\operatorname{span}\{\eta f: \eta \in V, f \in \mathrm{C}(T, \mathbb{K})\}$ is dense in $\mathrm{C}(T, V)$.

One can use the truncation procedure. From Lemma 2.8 (ii) it follows

$$
\lim _{c \rightarrow \infty} \sup _{t \in T}\left\|\xi_{t}^{(c)}-\xi_{t}\right\|=0
$$

hence for some $c>0$ it holds $\sup _{t \in T}\left\|\xi_{t}^{(c)}-\xi_{t}\right\|<\varepsilon / 3$. One constructs as in the proof of Theorem 2.4 using (F 8)

$$
\hat{\xi}_{t}^{(c)}:=\sum_{i=1}^{n} \xi_{t_{i}}^{(c)} z_{i}(t)
$$

with $\hat{\xi}^{c} \in \operatorname{span}\{\eta f: \eta \in V, \mathrm{P}(|\eta| \leq c)=1, f \in \mathrm{C}(T, \mathbb{K})\}$ and

$$
\sup _{t \in T}\left\|\hat{\xi}_{t}^{(c)}-\xi_{t}^{(c)}\right\|<\varepsilon / 3
$$

For finishing the proof it again remains to approximate the functions $z_{i} \in \mathrm{C}(T, \mathbb{K})$ by functions $\tilde{z}_{i}(t) \in A \subseteq \mathrm{C}(T, \mathbb{K})$ which is possible by the deterministic StoneWeierstrass Theorem 1.3. Based on (F 3) and (F5) on finds $\delta>0$ such that $\left\|\xi_{t_{i}}^{(c)} \Delta\right\|<\varepsilon /(3 n)$ for $i=1, \ldots, n$ and $\Delta \leq \delta$. Then for suitable $\tilde{z}_{i}(t) \in A \subseteq \mathrm{C}(T, \mathbb{K})$ with $\sup _{t \in T}\left|z_{i}(t)-\tilde{z}_{i}(t)\right|<\delta$ one gets

$$
\begin{gathered}
\sup _{t \in T}\left\|\xi_{t}-\sum_{i=1}^{n} \xi_{t_{i}}^{(c)} \tilde{z}_{i}(t)\right\| \\
\leq \sup _{t \in T}\left\|\xi_{t}-\xi_{t}^{(c)}\right\|+\sup _{t \in T}\left\|\xi_{t}^{(c)}-\hat{\xi}_{t}^{(c)}\right\|+\sum_{i=1}^{n} \sup _{t \in T}\left\|\hat{\xi}_{t_{i}}^{c}\left(z_{i}(t)-\tilde{z}_{i}(t)\right)\right\|<\varepsilon .
\end{gathered}
$$


Due to Lemma 2.9 this theorem includes the case of uniform stochastic convergence on $T$.

Theorem 2.11. If $T \subset \mathbb{R}^{d}\left(d \in \mathbb{N}^{*}\right)$ is a compact set, the additional properties ( $\left.\mathrm{F} 6\right)$ (F 8) of the F-norm in the previous theorem are not needed.

This is due to the fact that there exist open covers of $T$, such that each point of $T$ is an element of at most $2^{d}$ open sets from this cover. Analogous results can also be stated for periodic random functions.

Theorem 2.12. Theorem 2.5 and Theorem 2.6 remain true if one considers instead of scalar-valued random variables random variables with values in a separable Hilbert space $\mathbb{X}$ and corresponding $F$-normed subspaces of $\mathrm{L}^{0}(\mathbb{X})$.

This can be shown again by using norms instead of absolute values in the proofs. The truncation procedure can also be adapted. To see this remark that the set of distributions of the random variables of $\left(\xi_{t} ; t \in T\right)$ is a relatively compact set. Then by the Theorem of Prokhorov (see, e.g., Billingsley [1], Theorem 6.2) there exist for each $\varepsilon>0$ a compact set $K_{\varepsilon} \subset \mathbb{X}$ with $\mathrm{P}\left(\xi_{t} \in K_{\varepsilon}\right)>1-\varepsilon$ for all $t \in T$. This set can also be assumed to be convex. Then the orthogonal projection on this set can be used for the truncation procedure.

\section{Conclusions}

Several generalizations of the Stone-Weierstrass theorem about the possibility of approximation of certain continuous random functions on compact sets by some random functions from some subset are presented. Also the case of random variables in the space of continuous functions is considered.

\section{References}

[1] Billingsley, P., Convergence of Probability Measures, John Wiley \& Sons, New York, 1968.

[2] Bogachev, V.I., Measure Theory, Springer, Berlin, 2007.

[3] Dieudonné, D., Foundations of Modern Analysis, Academic Press, New York, 1969.

[4] Dugué, D., Traité de Statistique Théoretique et Appliquée, Masson, Paris, 1958.

[5] Onicescu, O., Istrăţescu, V.I., Approximation theorems for random functions, Rend. Mat. Serie VI, 8(1975), 65-81.

[6] Prolla, J.B., Weierstrass-Stone, the Theorem, Lang, Frankfurt am Main, 1993.

[7] Rolewicz, S., Metric Linear Spaces, D. Reidel Publishing Company, Dordrecht, 1984.

[8] Rudin, W., Real and Complex Analysis, McGraw-Hill Book Company, New York, 1970.

[9] Rudin, W., Functional Analysis, McGraw-Hill Book Company, New York, 1973.

[10] Ryabykh, V.G., Tokmakova,N.N., Yablonskiı̈, A.Ya., Approksimatsiya sluchä̌nykh funktsii (Approximation of random functions), In: Matematicheskii analiz i ego prilozheniya, Rostov na Donu, (1985), 131-135.

[11] Timofte, V., Stone-Weierstrass theorems revisited, J. Approx. Theory, 136(2005), 45-59. 
Hans-Jörg Starkloff

Technische Universität Bergakademie Freiberg

Faculty of Mathematics and Computer Sciences

Prüferstraße 9, D-09599 Freiberg, Germany

e-mail: Hans-Joerg. Starkloff@math.tu-freiberg.de 